\title{
Spontaneous Scaling Emergence in Generic Stochastic Systems
}

\author{
Sorin Solomon and Moshe Levy * \\ Racah Institute of Physics, Hebrew University, Jerusalem 91904, Israel
}

(21 August 1996)

We extend a generic class of systems which have previously been shown to spontaneously develop scaling (power law) distributions of their elementary degrees of freedom.

While the previous systems were linear and exploded exponentially for certain parameter ranges, the new systems fulfill nonlinear time evolution equations similar to the ones encountered in Spontaneous Symmetry Breaking (SSB) dynamics and evolve spontaneously towards "fixed trajectories" indexed by the average value of their degrees of freedom (which corresponds to the SSB order parameter). The "fixed trajectories" dynamics evolves on the edge between explosion and collapse/extinction.

The systems present power laws with exponents which in a wide range $(\alpha<-2$.) are universally determined by the ratio between the minimal and the average values of the degrees of freedom. The time fluctuations are governed by Levy distributions of corresponding power. For exponents $\alpha>-2$ there is no "thermodynamic limit" and the fluctuations are dominated by a few, largest degrees of freedom which leads to macroscopic fluctuations, chaos and bursts/intermitency.

\footnotetext{
*Email:sorin@vms.huji.ac.il, shiki@astro.huji.ac.il; http://shum.huji.ac.il/] sorin
} 
The wide-spread appearance of scaling laws in various natural systems has attracted the attention of scientists for the last century [1], [2], [3]. While for a measure zero set of systems (statistical mechanics systems in equilibrium at critical values of the parameters) scaling was thoroughly understood [4], [5], [6], for most of the scaling systems in nature, the origins of scaling remains a mystery [7]. It was felt for the last decade that in many cases the emergence [8] of scaling has to do with complexity and self organization, i.e. some systems have a tendency to organize spontaneously into critical states [9]. Moreover, the identification of the relevant degrees of freedom of a complex system was shown to be essential in understanding its multiscale dynamics 10] in terms of its "irreducibly complex cores" [11].

In a recent publication [12] it was shown that the appearance of the scaling power laws is as generic in multiplicative stochastic systems as the Boltzmann law is in additive stochastic systems. By using the logarithm of the normalized degrees of freedom, we showed that even non-stationary systems can be investigated by mapping them into additive-stochastic systems similar to usual statistical-mechanics systems in thermal equilibrium.

In the present paper we extend our mechanism to include a series of new features which makes it applicable to a wider range of phenomena.

In particular we explain the natural emergence of "fixed trajectories" for which, (as opposed to the economics example in [12]) the average mean value does not diverge in time.

These "fixed trajectories" are analog to the "fixed points" in equilibrium statistical mechanics systems and naturally evolve on the edge between exponential divergence and exponential decay.

We show that on these "fixed trajectories", the systems display power laws and Levy fluctuations distributions which have wide universality basins with computable exponents. 
For exponents $\alpha>-2$ of the Levy distributions, one expects the inexistence of a "thermodynamic limit" at large system sizes and the appearance of bursts and intermitency dominated by the fluctuations of the values of a few individual degrees of freedom (low dimensional chaos).

We call our generic mechanism Spontaneous Scaling Emergence (SSE) as it does not require criticality or self-organization to achieve the scaling state. The effective time evolution equations have common features with the equations of Spontaneous Symmetry Breaking.

Before presenting the extensions let us discuss from the novel point of view certain aspects of the systems introduced in [12]. These systems are characterized by the following stochastic time evolution equation :

$$
w_{i}(t+1)=\lambda w_{i}(t) ; \quad i=1,2, \ldots, N
$$

For definiteness one can think of the example of economic systems [12], where $w_{i}(t)$ represents the wealth of investor $i$ at time $t . \quad \lambda$ is a random variable drawn from a generic distribution $\Pi(\lambda, t)$. It was imposed in [12] that there is some lower bound to the value of $w_{i}(t)$ :

$$
w_{i}(t) \geq w_{0} \bar{w}(t) \quad \forall i, t
$$

where

$$
\bar{w}(t)=1 / N \sum_{i=1}^{n} w_{i}(t) .
$$

This dynamics was shown to lead to a power law distribution for the various $w$ values:

$$
\Phi(w, t)=C(t) w^{\alpha}
$$

where $C(t)$ is a normalization factor and $\alpha=-1-\frac{1}{1-w_{0}}$. For values of $w_{0}$ small compared to $1 / N$ (i.e. $-\log w_{0}>\log N$ ) there are corrections to (4) originating in the 
contributions of large $w$ values. The large $w$ corrections are relevant only for power laws with exponents $\alpha$ more positive than -2 and were discussed in [13]. For the sake of brevity we will not discuss them here again in the new context. One also expects a deviation from (4) in the immediate vicinity of the lower cut-off $w_{0} \bar{w}(t)$.

With these restrictions, the striking property of (田) is its independence on $\Pi(\lambda, t)$ except for the coefficient $C(t)$ which might depend on time in a quite irregular fashion.

Since we are looking here for systems in which $C(t)$ flows naturally and robustly to finite $C$ values, it is instructive to recall in some detail how the robustness of (4) arrised in [12]. The crucial step was to consider in place of the time-dependent distribution (四) the distribution of the normalized variables

$$
\omega(t)=w_{t} / \bar{w}(t)
$$

The distribution $P(\omega, t)$ of the variables $\omega(t)$ is "pinned" at least against overall rescalings $\omega \longrightarrow K(t) \omega$ by the condition

$$
\int \omega P(\omega, t) d \omega=1
$$

which follows trivially from the normalization (5). Equation (11) becomes in terms of the new variables:

$$
\omega_{i}(t+1)=\mu \omega_{i}(t)
$$

where the distribution $\Pi^{\prime}(\mu)$ of the random variable $\mu$ is related to the distribution $\Pi(\lambda)$ of $\lambda$ through the relation

$$
\Pi^{\prime}(\mu, t)=\Pi\left(\frac{\bar{\omega}(t)}{\bar{\omega}(t+1)} \mu, t\right) \frac{\bar{\omega}(t+1)}{\bar{\omega}(t)}
$$

This overall rescaling in the argument of $\Pi$ turned out to be enough to eliminate the dependence of $C$ on $t$ in (田). This means that the use of $\omega^{\prime} s$ in place of $w$ 's rescales in a very particular way the argument of $\Pi$. More precisely $\Pi^{\prime}(\mu, t)$ is fine-tuned by the transformation (5) such that $P(\omega, t+1)=P(\omega, t)$, i.e. (eq. 9 in $\operatorname{Ref}[12])$ 


$$
\int \Pi^{\prime}(\mu, t) \mu^{1+1 /\left(1-\omega_{0}\right)} d \mu=1
$$

This steady state situation is obviously reached by having the distribution of the $\mu^{\prime} s$ which appear in (更) finely tuned to equilibrate the values of $\mu$ larger than 1 (which lead to the increase of the $\omega$ 's) with the smaller $\mu$ values (which decrease the $\omega$ 's).

One sees now that one could in principle allow the shape of the distribution $\Pi(\lambda, t)$ to depend on $w$ as long as an overall rescaling of its parameter $\lambda \longrightarrow \frac{\bar{\omega}(t)}{\bar{\omega}(t+1)} \lambda$ insures that the distribution stability condition (9) holds for each $\omega$ i.e. $\int \Pi^{\prime}(\mu, t, \omega) \mu^{1+1 /\left(1-\omega_{0}\right)} d \mu=$ $1 \forall \omega, t$. In terms of the stock market this means that the power law is stable even if the spread (width) of the gain-loss distribution depends on the wealth of the investor (or varies in time). All that is required is that the average gain expectancies of all the investors are equal (except for the poorest, which can not lose more than allowed by the poverty bound $w_{0}$ ).

This is quite a large degree of universality even though unlike critical phenomena of statistical mechanics the scaling exponents depend here on a continuous parameter.

Now it is clear what is needed in order to get rid of the time dependence of $C(t)$ in (四): one needs to find a mechanism which brings $\Pi(\lambda)$ to be finely balanced between $\lambda>1$ and $\lambda<1$ values, such as to fulfill equation (9). Such a mechanism will prevent the exponential explosion or collapse of (1). In the stock market example this mechanism is expressed by the fact that as long as the total real wealth of the community is unchanged, overall gain factors across the entire population are irrelevant (they express just inflation in the instruments of payment). It will turn out that this is a degenerate example of a wider generic phenomenon.

In order to see how our new models exploit this idea, consider a system governed by the stochastic evolution rule :

$$
\begin{gathered}
\omega_{i}(t+1)-\omega_{i}(t)=\kappa\left(\omega_{i}(t)+\omega_{0}\right) ; \quad i=1,2, \ldots, N \\
\omega_{i}(t) \geq 0 \quad \forall i, t
\end{gathered}
$$


where $\kappa$ is a random variable drawn from a distribution $\Upsilon(\kappa, \bar{\omega}(t))$. We use different symbols for the analogues of $\lambda$ and $\Pi$ of (11) in order to avoid possible confusion. Moreover note that in the new models there is no normalization condition on the elementary variables $\omega_{i}(t)$ and therefore their average $\bar{\omega}(t)$ is a variable. In fact, $\Upsilon(\kappa, \bar{\omega}(t))$ depends on $\bar{\omega}(t)$ and is assumed that for $\bar{\omega}(t)=0$ the distribution $\Upsilon(\kappa, 0)$ favors the appearance of strongly positive values of $\kappa$ while for $\bar{\omega}(t)$ at a larger scale $W$, the distribution $\Upsilon(\kappa, W)$ favors negative values of $\kappa$. According to (10), this means that for small values of $\bar{\omega}(t)$ the $\omega^{\prime} s$ will have the tendency to increase while for $\bar{\omega}(t) \approx W$ they will have a tendency to decrease. This means that there exists a value $\bar{\omega}_{e q}$ (determined by a condition of the type (9) towards which $\bar{\omega}(t)$ will always flow, by the virtue of (10). (We defer to further publications the discussion of non-monotonic $\Upsilon$ 's which lead to multiple solutions $\bar{\omega}_{e q}$ and to possible branchings/bifurcations).

Once $\bar{\omega}_{e q}$ is reached, eq. (10) becomes of the type (7) (for the variable $\left.\omega_{i}(t)+\omega_{0}\right)$. Independently of the details of $\Upsilon(\kappa, \bar{\omega}(t))$ one then has :

$$
P(\omega)=C\left(\omega+\omega_{0}\right)^{-1-1 /\left(1-\omega_{0} / \bar{\omega}_{e q}\right)}
$$

with constant $C$. This is a stationary power law, similar in form to (四), but with no time dependence! According to the analysis in [12], the time fluctuations of the system are then characterized by a random walk with steps of variable stochastic length distributed according to (12). This means, according to the generalized central limit theorem, that the time fluctuations of various quantities are given by a Levy distribution [17] of corresponding power.

In the case in which the Levy distribution is wider (and the the power law exponent $\alpha$ is $>-2$. due to the effect of large $w_{i}$ values [13]), large macroscopic fluctuations are expected due to the variations of the large $w_{i}$ 's. In particular, they might bring a system macroscopically away from the fixed point $\bar{\omega}_{e q}$. This leads to a burst of non-equilibrium activity until enough correcting (relaxation) steps take place. In the case that it exists more then one $\bar{\omega}_{e q}$ value, these macroscopic fluctuations may induce 
jumps between the various "equilibrium" trajectories. The fact that for $\alpha>-2$ Levy distributions only the largest elements are relevant to the dynamics predicts the absence of a thermodynamic limit as the number of elementary degrees of freedom is taken to infinity. Such a phenomenon might explain some of the simulation results in [14], [15]. We propose to study these effects numerically and compare with the theoretical and experimental universal properties of chaotic systems [16].

The assumption in (10) that the distribution $\Upsilon(\kappa, \bar{\omega}(t))$ depends on $\bar{\omega}(t)$ might seem arbitrary, but in fact it arises naturally in quite familiar contexts. Consider a generic complex system in which some cooperative "short-range" underlying dynamics insures that certain subsystems act coherently and can be represented by a single variable $\omega_{i}(t)$. One can think of such subsystems as families, clans, populations within an ecology, etc. Assume that isolated from each other these systems act according to (1). Suppose however that there is an "infinite-range" competitive interaction between the $\omega$ 's (clans competing for land, populations for food, etc.). The resulting dynamics may then be of the form

$$
\omega_{i}(t+1)-\omega_{i}(t)=\kappa \omega_{i}(t)+\omega_{0} \bar{\omega}(t)-\frac{a}{2} \bar{\omega}(t) \omega_{i}(t)+\text { etc. },
$$

where etc. stands for possible additional inhomogeneous terms. One sees that for small values of $\bar{\omega}$ the stochastic equation (13) drives the $\omega$ 's to higher values while for large values of $\bar{\omega}$ the $\omega$ 's are driven to smaller values and one is in the case (10).

Note that equation (13) is very similar to the familiar equation governing the emergence of Spontaneous Symmetry Breaking (SSB):

$$
\dot{M}=\kappa M-\frac{a}{2} M^{2}+\text { etc. }
$$

from which it differs only by the fact that it mixes the "short-range" variable $\omega_{i}(t)$ with the "infinite-range" variable $\bar{\omega}(t)$.

This suggests the extension of our models for "vectorial" $\omega$ variables which take values in spaces with various symmetries. 
In terms of the SSB vocabulary, the power of the distribution (12) is governed by the ratio between the scale of the global variable $\bar{\omega}(t)$ and the scale of the local variable $\omega_{0}$. As seen above, both of them may be dynamically fixed by the interplay of various non-homogeneous terms in (13). Note that the emergence of power laws and hierarchies follows then naturally even if the ratio of the 2 scales is of order 1 (e.g. $0.2-0.3$ in the economics application [12]).

In further studies one can consider the effects of higher powers in (13) and relax the "infinite-range" assumption. In fact, one can study multiscale hierarchies of ranges, and consider the domains of almost constant $\omega$ 's as the elementary degrees of freedom $\Omega$ of a coarser system. For instance, each family within a clan acts as a unit but competes with the other families for the clans resources. Yet at a larger scale, the clan acts as a self-reinforcing unit in competition with other clans over the country resources, and so on. Examples in diluted finite connectivity spin glasses [11] and Weiss domains in magnetic systems may be of relevance.

This multiscale cooperation-competition interplay might be particularly relevant for the study of the speciation process in various ecological and territorial conditions. It also raises some suggestions on the ways to implement the reductionist program as one passes from one scale to another. The scaling laws and their relevant degrees of freedom might give some objective criterion for identifying the passage from one level of description to another. In the spirit of [10] and [11] one can "turn the tables" and use the power laws in order to identify from (12) the size and identity of the relevant effective degrees of freedom of the system: the "irreducibly complex cores" in the terminology of [11]. In turn, the dynamics of $\bar{\omega}$ would indicate the "multiscale-complex" dynamics of the hierarchy of macros in the terminology of [10].

Let us reiterate in conclusion the main points discussed in the present paper.

1. We have shown that systems with local self-catalizing multiplicative interactions and competitive global interactions (10) (13), may generically flow into "fixed 
trajectories" at the edge between explosion and collapse.

2. The various "fixed trajectories" are indexed by the values of the average degrees of freedom which emerge from a nonlinear time evolution equation similar in form to the one governing SSB.

3. In those equations, both local and global variables appear and their scales $\left(\omega_{0}\right.$ and $\bar{\omega}$ ) may be fixed by the generic nonlinear dynamics.

4. The degrees of freedom are distributed according power laws (12) whose exponents are determined universally (for a wide range) only by the ratio of the 2 scales $\omega_{0} / \bar{\omega}$.

5. The fluctuations around the "fixed trajectories" are governed by Levy distributions of corresponding (universal) exponents.

6. In the cases in which the Levy distribution has exponent $\alpha>-2$ the system does not have a thermodynamic limit and presents bursts and intermitency produced by the few degrees of freedom with the largest values (chaos [16]).

We are now facing the enormous task of applying the present framework to particular complex systems beyond the economic example presented in [12]. The most urgent objective is to relate for each system the complex dynamics to the appropriate $\omega$ 's, identify $\omega_{0}$ and relate it by (12) to the actual power law measured experimentally.

The values of $\omega_{0}$ may be determined by different mechanisms in various systems. Examples for such mechanisms are the extinction of species with too few members, subsidies to the poor, minimal size of population needed to found a city, discretization, additive noise, pumping of energy, etc. 
[1] Pareto, V. Cours d'Economique Politique, Vol 2, (1897).

[2] Zipf, G. K. Human Behavior and the Principle of Least Effort (Addison Wesley, Cambridge MA, 1949).

[3] Mandelbrot, B. B. Comptes Randus 232, (1951) 1638-1640.

[4] Kadanoff, L. Physics2, (1966) 263.

[5] Fisher, M. E. Reviews of Modern Physics46, (1974) 597.

[6] Wilson, K. G. Reviews of Modern Physics47, (1975) 773.

[7] Gell-Mann, M. The Quark and the Jaguar, 92-106 (Little Brown and Co., London, 1994).

[8] Stuart A Kauffman, At home in the universe, Oxford University Press, New York 1995,

[9] Bak, P., Tang, C. \& Wiesenfeld, K. Phys. Rev. Lett. 59, (1987) 381.

[10] S. Solomon, The Microscopic Representation of Complex Systems, in Annual Rev. of Comput. Phys. II, ed. D. Stauffer, World Scientific, Singapore 1995.

[11] Persky, N and Solomon, S. Phys. Rev. E. (1996) to appear.

[12] Levy, M., and Solomon, S. Intn. J. Mod. Phys. C 7, No. 4 (1996) 595.

[13] Levy, M., and Solomon, S. Intn. J. Mod. Phys. C 7, No. 1 (1996) 65.

[14] M. Levy, N. Persky and S. Solomon, Int. J. High Speed Computing to appear (1966).

[15] T. Hellthaler, Int. J. Mod. Phys. C 6 (1995) 845.

[16] M. J. Fiegenbaum J. Stat. Phys. 1925 (1978) 669.

E. N. Lorentz J. Atmos. Sci. 20 (1963) 130. 
R. M. May Nature 261 (1976) 459 .

[17] P. Levy, Theorie de l'Addition des Variables Aleatoires (Gauthier-Villiers, Paris, 1937).

Note Added in Proof

A detailed discussion of some generalizations of the model introduced in [12] can be found in "Convergent Multiplicative Processes Repelled from Zero" by D. Sornette and R. Cont. We thank D. Sornette for very instructive correspondence. We thank D. Stauffer for advice and encouragement since the beginning of our research on the present subject. 